Faculty of Mathematical Sciences

\section{University of Twente}

University for Technical and Social Sciences
P.O. Box 217

7500 AE Enschede

The Netherlands

Phone: +31-53-4893400

Fax: +31-53-4893114

Email: memo@math.utwente.nl

Memorandum No. 1558

(Average-) convexity of common

pool and oligopoly TU-games

T.S.H. Driessen and H. Meinhardt ${ }^{1}$

DeCember 2000

ISSN 0169-2690

\footnotetext{
${ }^{1}$ Projekt Umweltgemeingüter, Lehrstuhl für Volkswirtschaftslehre 3, Postfach 69 80, Universität Karlsruhe, Zirkel 2, D-76128 Karlsruhe, Germany
} 


\title{
(AVERAGE-) CONVEXITY of COMMON POOL and OLIGOPOLY TU-GAMES *
}

\author{
Theo DRIESSEN ${ }^{\dagger} \quad$ Holger MEINHARDT ${ }^{\ddagger}$
}

\begin{abstract}
The paper studies both the convexity and average-convexity properties for a particular class of cooperative TU-games called common pool games. The common pool situation involves a cost function as well as a (weakly decreasing) average joint production function. Firstly, it is shown that, if the relevant cost function is a linear function, then the common pool games are convex games. The convexity, however, fails whenever cost functions are arbitrary. We present sufficient conditions involving the cost functions (like weakly decreasing marginal costs as well as weakly decreasing average costs) and the average joint production function in order to guarantee the convexity of the common pool game. A similar approach is effective to investigate a relaxation of the convexity property known as the average-convexity property for a cooperative game. An example illustrates that oligopoly games are a special case of common pool games whenever the average joint production function represents an inverse demand function.
\end{abstract}

Keywords: common pool situation, cooperative TU-game, common pool TU-game, oligopoly TU-game, convexity, average-convexity

1991 Mathematics Subject Classifications: 90D12, 90D40

\section{Introduction and background}

The "tragedy of the commons" is a well-known phenomenon throughout the exhaustive literature on common pool resources. According to the solution part of non-cooperative game theory (i.e., pure Nash equilibria), the common pool resources are overused; in other words, the commonly owned lake is overfished by the society of fishermen and the tragedy of the commons occurs (cf. [4], [11], [9]). In order to avoid the tragedy of the commons, one may focus on a (partially) cooperative game theoretic approach to the common pool situation, in which cooperation among fishermen is assumed to some extent. For that purpose, the partition function form (or coalition structure) approach was treated in [3], which deals with the non-cooperative game solution (i.e., Nash equilibrium for a suitably chosen game in normal form) as well as the cooperative game solution (i.e., (non)existence of core allocations for two types of appropriately chosen cooperative TU-games). In this paper we deal with a

\footnotetext{
*The research for this paper was partially done during a stay (September 1999) of the second author at the Faculty of Mathematical Sciences, University of Twente, Enschede, The Netherlands. This paper has been presented by the first author at the International Conference on Operations Research and Game Theory (ICORGT-2000) held at Chennai (IIT Madras), India, January 3-7, 2000

${ }^{\dagger}$ Theo S.H. Driessen, Faculty of Mathematical Sciences, University of Twente, P.O. Box 217, 7500 AE Enschede, The Netherlands. E-mail: t.s.h.driessen@math.utwente.nl

${ }^{\ddagger}$ Holger Meinhardt, Projekt Umweltgemeingüter, Lehrstuhl für Volkswirtschaftslehre 3, Postfach 6980 , Universität Karlsruhe, Zirkel 2, D-76128 Karlsruhe, Germany. E-mail: hme@vwl3.wiwi.uni-karlsruhe.de
} 
fully cooperative game theoretic approach to the common pool situation, following the overall treatment in [6], [7], [8]. Our treatment is fully based on the so-called common pool cooperative TU-game, which arises directly from the underlying normal form game by applying the standard maxmin-technique. The main goal of this paper is to study the convexity property (and related notions) for this common pool TU-game, in which different types of cost functions are investigated to guarantee the convexity of the underlying game. In the field of cooperative game theory, the convexity of a game is an extremely appealing feature in order to determine various solution concepts (like the existence, structure and largeness of the core). Let the model of an economy with a common pool resource be described by a society of fishermen, denoted by the finite set $N:=\{1,2, \ldots, n\}$, who are employed on a commonly owned lake. For any fisherman $i \in N$, let $x_{i} \geq 0$ represent the amount of labour that $i$ expends to catch fish. Clearly, the overall amount of labour is given by $\sum_{j \in N} x_{j}$. The relevant technology that determines the amount of fish caught is considered to be a function of the overall amount of labour, called the joint production function $f: \mathbb{R}_{+} \rightarrow \mathbb{R}_{+}$satisfying $f(0)=0$. The distribution of fish among fishermen is supposed to be proportional to the amount of labour expended by individual fishermen. In other words, the amount of fish assigned to fisherman $i$ is given by $\frac{x_{i}}{\sum_{j \in N} x_{j}} \cdot f\left(\sum_{j \in N} x_{j}\right)$. The price of fish is normalized to be one unit of money and let $c: \mathbb{R}_{+} \rightarrow \mathbb{R}_{+}$denote an arbitrarily chosen cost function of labour satisfying $c(0)=0$. Generally speaking, the cost function includes salary costs (e.g., unit price of labour in case of a linear cost function) and, if it applies (in case of non-linear cost functions), taxes, social security and insurance costs, and so on, due to the labour input.

Due to the non-cooperative game theoretic approach, the common pool economy is modelled as a (non-cooperative) game in normal form $\Gamma=\left\langle X_{1}, X_{2}, \ldots, X_{n}, F_{1}, F_{2}, \ldots, F_{n}\right\rangle$, where the player set $N=\{1,2, \ldots, n\}$ represents the society of fishermen, and, for any fisherman $i \in N$, a strategy $x_{i} \in X_{i}$ of the strategy set $X_{i} \subseteq \mathbb{R}_{+}$represents the amount of labour by $i$ and the netto income function $F_{i}: X_{1} \times X_{2} \times \ldots \times X_{n} \rightarrow \mathbb{R}$ is given by

$F_{i}\left(\left(x_{k}\right)_{k \in N}\right):=\frac{x_{i}}{\sum_{j \in N} x_{j}} \cdot f\left(\sum_{j \in N} x_{j}\right)-c\left(x_{i}\right) \quad$ for all $i \in N$, all $x_{k} \in X_{k}, k \in N$.

Throughout the paper, it is supposed that every fisherman $i \in N$ is initially endowed with labour the amount of $w_{i} \geq 0$ and thus, the strategy set $X_{i}$ of player $i$ equals the interval $\left[0, w_{i}\right]:=\left\{y \in \mathbb{R} \mid 0 \leq y \leq w_{i}\right\}$. In accordance with the solution part of non-cooperative game theory, every fisherman $i$ will choose his labour input $x_{i}^{*}$ to maximize his own netto income $F_{i}\left(x_{i},\left(x_{k}^{*}\right)_{k \in N \backslash\{i\}}\right), x_{i} \in\left[0, w_{i}\right]$, given the labour inputs $\left(x_{k}^{*}\right)_{k \in N \backslash\{i\}}$ of the other fishermen $k, k \in N \backslash\{i\}$. Under certain circumstances (like linearity of the cost function with marginal constant cost $c$ and strict concavity of the joint production function $f$ in that $f^{\prime \prime}<0$ and $f^{\prime}>0$ ), there exists a unique non-cooperative game solution called Nash equilibrium. The main result, however, states that the overall amount of labour inputs by the Nash equilibrium $\left(x_{k}^{*}\right)_{k \in N}$ exceeds the Pareto efficient level (or equivalently, the social optimum) $x^{*}$, that is $\sum_{k \in N} x_{k}^{*}>x^{*}$. The social optimum $x^{*}$ is implicitly determined by the unique solution of the joint maximization problem $f(x)-c \cdot x, x \geq 0$, where the constant $c \geq 0$ denotes the marginal cost of the linear cost function. In short, the commonly owned lake is overfished by the fishermen and the tragedy of the commons occurs.

As mentioned before, this paper is devoted to a cooperative game theoretic approach to the common pool economy. Our first task is to transform the (non-cooperative) game in normal form $\Gamma$, as given by (1.1), into a so-called cooperative game (with transferable utility) and this transformation is fully based on the known maxmin-technique. In order to define the 
characteristic function $v: \mathcal{P}(N) \rightarrow \mathbb{R}$ on the power set $\mathcal{P}(N):=\{S \mid S \subseteq N\}$ of the player set $N$, let the worth $v(S)$, for every coalition $S \subseteq N$, arise from the two-person non-cooperative setting in which coalition $S$ is confronted with its complementary coalition $N \backslash S$ in such a way that members of $S$ aim to maximize their "worst" cases. In other words, for every coalition $S \subseteq N, S \neq \emptyset$, the members of $S$ will choose their own individual strategies $\left(x_{k}\right)_{k \in S}$ to maximize the worst case in that the opponents of $S$ choose their own individual strategies $\left(x_{k}\right)_{k \in N \backslash S}$ such that the overall netto income $\sum_{j \in S} F_{j}\left(\left(x_{k}\right)_{k \in N}\right)$ of coalition $S$ is minimal (given the strategies by members of $S$ ). Under the assumption of the linearity of the joint cost function with marginal constant cost $c$, the induced cooperative $T U$-game $\langle N, v\rangle$ assigns to every coalition $S \subseteq N, S \neq \emptyset$, the following worth:

$$
\begin{aligned}
v(S) & :=\max _{\left(x_{k}\right)_{k \in S} \in\left(X_{k}\right)_{k \in S}} \min _{\left(x_{k}\right)_{k \in N \backslash S} \in\left(X_{k}\right)_{k \in N \backslash S}} \sum_{j \in S} F_{j}\left(\left(x_{k}\right)_{k \in N}\right) \\
& =\max _{\left(x_{k}\right)_{k \in S} \in\left(X_{k}\right)_{k \in S}} \min _{\left(x_{k}\right)_{k \in N \backslash S} \in\left(X_{k}\right)_{k \in N \backslash S}}\left[\left[\sum_{j \in S} x_{j}\right] \cdot \frac{f\left(\sum_{j \in N} x_{j}\right)}{\sum_{j \in N} x_{j}}-c \cdot\left[\sum_{j \in S} x_{j}\right]\right] \\
& \left.=\max _{y} \min _{z}\left[y \cdot \frac{f(y+z)}{y+z}-c \cdot y\right] \quad \text { (here } y:=\sum_{j \in S} x_{j} \text { and } z:=\sum_{j \in N \backslash S} x_{j}\right) \\
& =\max _{0 \leq y \leq w_{S}} \min _{0 \leq z \leq w_{N \backslash S}}[y \cdot h(y+z)-c \cdot y]
\end{aligned}
$$

By taking into account the individual endowments $w_{i}, i \in N$, it is supposed that the overall endowments of the members of any coalition $T, T \subseteq N$, is simply obtained by addition (additive sum), denoted as $w_{T}:=\sum_{j \in T} w_{j}$. This additional assumption elucidates why the restrictions $0 \leq y \leq w_{S}$ and $0 \leq z \leq w_{N \backslash S}$ respectively appear in (1.2). Moreover, the average joint production function $h: \mathbb{R}_{+} \rightarrow \mathbb{R}_{+}$is given by $h(x):=\frac{f(x)}{x}$ for all $x>0$. The standard assumption of concavity of the joint production function $f$ (i.e., $f^{\prime \prime} \leq 0$ ) implies that the average joint production function $h$ is weakly decreasing (i.e., $h^{\prime} \leq 0$ ). Thus, for every coalition $S \subseteq N, S \neq \emptyset$, the minimization problem in (1.2) is solved for $z=w_{N \backslash S}$, i.e., each opponent $j \in N \backslash S$ invests his total endowment $w_{j}$ to minimize the overall netto income to the coalition $S$ given their own (overall) strategy $y, 0 \leq y \leq w_{S}$. In summary, the characteristic function $v: \mathcal{P}(N) \rightarrow \mathbb{R}$ of the common pool TU-game $\langle N, v\rangle$ (with respect to a linear cost function with marginal cost $c$ ) is given by $v(\emptyset):=0$ and

$$
v(S):=\max _{0 \leq y \leq w_{S}}\left[y \cdot h\left(y+w_{N \backslash S}\right)-c \cdot y\right] \quad \text { for all } S \subseteq N, S \neq \emptyset .
$$

The common pool TU-game $\langle N, v\rangle$ is said to be convex (or supermodular) if its characteristic function $v: \mathcal{P}(N) \rightarrow \mathbb{R}$, as given by (1.3), satisfies one of the following two equivalent conditions (cf. [10], [1]):

$$
\begin{array}{rlrl}
v(S)+v(T) & \leq v(S \cup T)+v(S \cap T) & & \text { for all } S, T \subseteq N, \text { or equivalently } \\
v(S \cup\{i\})-v(S) & \leq v(T \cup\{i\})-v(T) & \text { whenever } S \subseteq T \subseteq N \backslash\{i\} .
\end{array}
$$

The main result of this paper (cf. the forthcoming Theorem 3.1) states that, without any further assumption on the weakly decreasing (average joint production) function $h$, the common 
pool TU-game $\langle N, v\rangle$ (with respect to a linear cost function) is a convex game. In addition, its tricky, but elegant proof turns out to be extremely helpful to investigate the convexity (and a related notion called average convexity) of common pool games with respect to non-linear cost functions.

\section{Two examples of Common Pool Games, e.g. oligopoly games}

Definition 2.1. Let $N$ be a finite set of individuals (players) and let $w_{i} \geq 0$ denote player $i$ 's endowment. For any $T \subseteq N$, denote the total of its members' endowments by $w_{T}:=\sum_{j \in T} w_{j}$, where $w(\emptyset):=0$. With any joint production function $f: \mathbb{R}_{+} \rightarrow \mathbb{R}_{+}$satisfying $f(0)=0$ as well as concavity (i.e., $f^{\prime \prime}(x) \leq 0$ for all $x>0$ ), there is associated its weakly decreasing average joint production function $h: \mathbb{R}_{+} \rightarrow \mathbb{R}_{+}$, given by $h(x):=\frac{f(x)}{x}$ for all $x>0$. Further, let $c: \mathbb{R}_{+} \rightarrow \mathbb{R}_{+}$be an arbitrarily twice-differentiable cost function satisfying $c(0)=0$.

The corresponding common pool cooperative TU-game $\langle N, v\rangle$ is defined in such a way that its player set $N$ consists of the users of the common pool resource and its characteristic function $v: \mathcal{P}(N) \rightarrow \mathbb{R}$ is given by $v(\emptyset):=0$ and

$$
v(S):=\max _{0 \leq y \leq w_{S}}\left[y \cdot h\left(y+w_{N \backslash S}\right)-c(y)\right] \quad \text { for all } S \subseteq N, S \neq \emptyset .
$$

Before we start to discuss two examples of common pool TU-games, we remark, without going into details, that the common pool game $\langle N, v\rangle$ of $(2.1)$ is a monotonic game, i.e., $v(T) \geq v(S)$ for all $S \subseteq T \subseteq N$ or equivalently, $v(S \cup\{i\}) \geq v(S)$ for all $i \in N$ and all $S \subseteq N \backslash\{i\}$. Particularly, $v(S \cup\{i\})=v(S)$ for all $S \subseteq N \backslash\{i\}$ whenever $w_{i}=0$. In words, a player with no initial endowment at all is a dummy player in the common pool game $\langle N, v\rangle$ of (2.1). Consequently, the convexity condition (1.4) in which a dummy player $i$ is involved (determined by $w_{i}=0$ ) is trivially satisfied by a common pool game. Generally speaking, it is supposed that any player possesses a positive endowment.

Example 2.2. (common pool games versus oligopoly games; cf. [12])

In the economic setting of an oligopoly market with $n$ firms producing a homogeneous good, let $w_{i} \geq 0$ represent the production capacity of firm $i$, denote the corresponding cost function by $c: \mathbb{R}_{+} \rightarrow \mathbb{R}_{+}$and let $h: \mathbb{R}_{+} \rightarrow \mathbb{R}_{+}$represent a weakly decreasing inverse demand function. For any $S \subseteq N, S \neq \emptyset$, the profit function $\pi_{S}: \mathbb{R}_{+} \rightarrow \mathbb{R}_{+}$for coalition $S$ is given by $\pi_{S}(y):=y \cdot h\left(y+w_{N \backslash S}\right)-c(y)$ for all $y \geq 0$, where the variable $y$ refers to the production level of coalition $S$ (assuming all the opponents of $S$ produce full capacity). In other words, common pool TU-games reduce to oligopoly games as soon as the average joint production function can be interpreted as some inverse demand function.

As a particular example of common pool games, let the average joint production function $h$ be an inverse demand function of the form $h(x):=\max [0, a-x]$ for all $x>0$. In other words, the underlying joint production function $f$ is given by a quadratic function, namely $f(x):=x \cdot(a-x)$ for all $0 \leq x \leq a$ and $f(x):=0$ for all $x \geq a$. Most important, $h$ is a weakly decreasing function. In addition, suppose that the cost function $c$ is a quadratic function of the form $c(y):=c_{2} \cdot y^{2}+c_{1} \cdot y$ for all $y \geq 0$ and certain constants $c_{2} \geq 0$, $0 \leq c_{1} \leq a$ (notice that a linear cost function arises whenever $c_{2}=0$ ). Our aim is to determine the associated common pool game $\langle N, v\rangle$ of $(2.1)$. For any coalition $S \subseteq N, S \neq \emptyset$, the maximization problem (2.1) with respect to coalition $S$ involves the maximization of 
the objective function $g_{S}(y):=y \cdot\left[a-c_{1}-w_{N \backslash S}-\left(1+c_{2}\right) \cdot y\right]$ under the two constraints $0 \leq y \leq w_{S}$ and $y \leq a-w_{N \backslash S}$. Generally speaking, the unconstrained maximization problem involving the objective quadratic function $g_{S}(y)$ attains its maximum for $y=\alpha_{S}$, where $\alpha_{S}:=\frac{a-c_{1}-w_{N \backslash S}}{2 \cdot\left(1+c_{2}\right)}$. Note that $g_{S}(y)=\left(1+c_{2}\right) \cdot y \cdot\left[2 \cdot \alpha_{S}-y\right]$ for all $y \geq 0$.

Obviously, if $\alpha_{S} \leq 0$, then the maximizer $y_{S}$ of the maximization problem (2.1) equals zero, thus $y_{S}=0$ and $v(S)=0$. In case $\alpha_{S}>0$, then the maximizer equals $\alpha_{S}$ or $w_{S}$ or $a-w_{N \backslash S}$, whichever is less. For the moment, suppose $w_{S} \leq a-w_{N \backslash S}$ or equivalently, $w_{N} \leq a$. To conclude with, if $0 \leq \alpha_{S} \leq w_{S}$, then $y_{S}=\alpha_{S}$ and thus, $v(S)=g_{S}\left(\alpha_{S}\right)=\left(1+c_{2}\right) \cdot\left(\alpha_{S}\right)^{2}$. If $\alpha_{S} \geq w_{S}$, then $y_{S}=w_{S}$ and thus, $v(S)=g_{S}\left(w_{S}\right)=\left(1+c_{2}\right) \cdot w_{S} \cdot\left[2 \cdot \alpha_{S}-w_{S}\right]$. In summary, we arrive at the following results concerning the maximizer $y_{S}$ as well as the worth $v(S)$ for any nonempty coalition $S$ (provided $w_{N} \leq a$ ):

$$
\begin{aligned}
& y_{S}= \begin{cases}0 & \text { if } \alpha_{S} \leq 0, \\
\alpha_{S} & \text { if } 0 \leq \alpha_{S} \leq w_{S}, \\
w_{S} & \text { if } \alpha_{S} \geq w_{S} ;\end{cases} \\
& v(S)= \begin{cases}0 & \text { if } \alpha_{S} \leq 0, \\
\left(1+c_{2}\right) \cdot\left(\alpha_{S}\right)^{2} & \text { if } 0 \leq \alpha_{S} \leq w_{S}, \\
\left(1+c_{2}\right) \cdot w_{S} \cdot\left[2 \cdot \alpha_{S}-w_{S}\right] & \text { if } \alpha_{S} \geq w_{S} ;\end{cases}
\end{aligned}
$$

In terms of the players' endowments $w_{i}, i \in N$, the intercept $a$ in the inverse demand function, and the cost figures $c_{1}$ and $c_{2}$, we present the worth $v(S)$ of $(2.3)$ as follows (provided $w_{N} \leq a$ ):

$$
v(S)= \begin{cases}0 & \text { if } \alpha_{S} \leq 0, \text { i.e., } w_{S} \leq w_{N}-\left(a-c_{1}\right), \\ {\left[a-c_{1}-w_{N}\right] \cdot w_{S}-c_{2} \cdot\left(w_{S}\right)^{2}} & \text { if } \alpha_{S} \geq w_{S}, \text { i.e., } w_{S} \leq \frac{a-c_{1}-w_{N}}{1+2 \cdot c_{2}}, \\ \frac{\left[a-c_{1}-w_{N \backslash S}\right]^{2}}{4 \cdot\left(1+c_{2}\right)} & \text { otherwise. }\end{cases}
$$

Proposition 2.3. In the context of Example 2.2, suppose that the overall amount of endowment $w_{N}$ exceeds the intercept $a$ in the inverse demand function, i.e., the overuse condition $w_{N} \geq a$. Then the common pool (or oligopoly) game $\langle N, v\rangle$ of (2.4) reduces as follows: $v(\emptyset)=0$ and

$$
v(S)=\frac{\left[\max \left[0, a-c_{1}-w_{N \backslash S}\right]\right]^{2}}{4 \cdot\left(1+c_{2}\right)} \quad \text { for all } S \subseteq N, S \neq \emptyset .
$$

Further, the common pool (or oligopoly) game $\langle N, v\rangle$ of (2.5) is a convex game (i.e., (1.4) holds since it is a positive multiple of the square of a so-called bankruptcy game, the characteristic function of which, in turn, is known to be convex as well as monotonic).

Proof. Suppose $w_{N} \geq a$. Clearly, for any $S \subseteq N, S \neq \emptyset$, it holds that $a-w_{N \backslash S} \leq w_{S}$ and moreover, if $\alpha_{S}>0$, then $\alpha_{S}=\frac{a-c_{1}-w_{N \backslash S}}{2 \cdot\left(1+c_{2}\right)}<a-c_{1}-w_{N \backslash S} \leq a-w_{N \backslash S}$ as well as $v(S)=g_{S}\left(\alpha_{S}\right)=\left(1+c_{2}\right) \cdot\left(\alpha_{S}\right)^{2}=\frac{\left[a-c_{1}-w_{N \backslash S}\right]^{2}}{4 \cdot\left(1+c_{2}\right)}$. This proves $(2.5)$. Define the characteristic function $u_{E, w}: \mathcal{P}(N) \rightarrow \mathbb{R}$ of the so-called bankruptcy $T U$-game $\left\langle N, u_{E, w}\right\rangle$ by $u_{E, w}(\emptyset):=0$ and $u_{E, w}(S):=\max \left[0, E-w_{N \backslash S}\right]$ for all $S \subseteq N, S \neq \emptyset$. Here the estate $E:=a-c_{1}$, whereas 
the claims of the claimants (players) are identified with their endowments. For convenience' sake, write $u$ instead of $u_{E, w}$. Since $v(S)=\frac{(u(S))^{2}}{4 \cdot\left(1+c_{2}\right)}$ for all $S \subseteq N$, the common pool (or oligopoly) game $\langle N, v\rangle$ of (2.5) is a positive multiple of the square $\left\langle N, u^{2}\right\rangle$ of the bankruptcy game $\langle N, u\rangle$. It is well-known that bankruptcy games are convex as well as monotonic (cf. [1]). Due to the convexity and monotonicity of the bankruptcy game $\langle N, u\rangle$, we conclude that its square $\left\langle N, u^{2}\right\rangle$ is a convex game too. Indeed, for all $i \in N$ and all $S \subseteq T \subseteq N \backslash\{i\}$, the following chain of (in)equalities holds:

$$
\begin{aligned}
& {[u(S \cup\{i\})]^{2}-[u(S)]^{2} } \\
= & {[u(S \cup\{i\})-u(S)] \cdot[u(S \cup\{i\})+u(S)] } \\
\leq & {[u(T \cup\{i\})-u(T)] \cdot[u(S \cup\{i\})+u(S)] \quad \text { (by convexity of the game }\langle N, u\rangle) } \\
\leq & {[u(T \cup\{i\})-u(T)] \cdot[u(T \cup\{i\})+u(T)] \quad \text { (by monotonicity of the game }\langle N, u\rangle) } \\
= & {[u(T \cup\{i\})]^{2}-[u(T)]^{2} }
\end{aligned}
$$

This completes the full proof of the convexity statements.

Proposition 2.4. In the context of Example 2.2, suppose that the overall amount of endowment $w_{N}$ is bounded above by $\frac{a-c_{1}}{2 \cdot\left(1+c_{2}\right)}$, i.e., the no-overuse condition $w_{N} \leq \frac{a-c_{1}}{2 \cdot\left(1+c_{2}\right)}$. Then the common pool (or oligopoly) game $\langle N, v\rangle$ of (2.4) reduces as follows: $v(\emptyset)=0$ and

$$
v(S)=\left[a-c_{1}-w_{N}\right] \cdot w_{S}-c_{2} \cdot\left(w_{S}\right)^{2} \quad \text { for all } S \subseteq N, S \neq \emptyset .
$$

In words, the common pool (or oligopoly) game $\langle N, v\rangle$ of (2.6) is the sum of an non-negative multiple of an additive game (arising from the players' endowments) and a non-positive multiple of the square of this additive game. Hence, $\langle N, v\rangle$ is a so-called concave game in that

$$
v(T \cup\{i\})-v(T) \leq v(S \cup\{i\})-v(S) \quad \text { whenever } S \subseteq T \subseteq N \backslash\{i\} .
$$

In case $c_{2}>0$ (i.e., a non-linear cost function) and $w_{k}>0$ for all $k \in N$, the inequality in (2.7) is strict and thus, the common pool (or oligopoly) game $\langle N, v\rangle$ of (2.6) fails to be convex (under the given circumstances with reference to a non-linear cost function).

Proof. Suppose $w_{N} \leq \frac{a-c_{1}}{2 \cdot\left(1+c_{2}\right)}$. Clearly, for any $S \subseteq N, S \neq \emptyset$, it holds that $w_{S} \leq a-w_{N \backslash S}$ (due to $w_{N} \leq a-c_{1} \leq a$ ) and moreover, $w_{S} \leq w_{N} \leq \frac{a-c_{1}-w_{N}}{1+2 \cdot c_{2}}$ or equivalently, $\alpha_{S} \geq w_{S}$. Thus, $v(S)=g_{S}\left(w_{S}\right)=\left[a-c_{1}-w_{N}\right] \cdot w_{S}-c_{2} \cdot\left(w_{S}\right)^{2}$ for all $S \subseteq N, S \neq \emptyset$. This proves (2.6). From (2.6), we derive that, for all $i \in N$ and all $S \subseteq N \backslash\{i\}$, the following equality holds:

$$
v(S \cup\{i\})-v(S)=\left[a-c_{1}-w_{N}\right] \cdot w_{i}-c_{2} \cdot\left(w_{i}\right)^{2}-2 \cdot c_{2} \cdot w_{i} \cdot w_{S}
$$

In view of (2.8), the concavity condition $v(T \cup\{i\})-v(T) \leq v(S \cup\{i\})-v(S)$ is equivalent to $c_{2} \cdot w_{i} \cdot w_{T} \geq c_{2} \cdot w_{i} \cdot w_{S}$ (provided $S \subseteq T$ ). The latter (weak) inequality holds true because the assumption $S \subseteq T$ yields $w_{T} \geq w_{S}$. This proves (2.7). In case $c_{2}>0$ and $w_{k}>0$ for all $k \in N$, then the strict inequality $w_{T}>w_{S}$ holds whenever $S \varsubsetneqq T$ and thus, the convexity condition (1.4) fails to hold under these circumstances (with reference to a non-linear cost function). This completes the full proof of the concavity and convexity statements. 
Remark 2.5. Oligopoly TU-games were studied in [12] with reference to a particular inverse demand function of the form $h(x):=\max [0, a-x]$ for all $x>0$ as well as linear costs functions with marginal costs $c_{i}, i \in N$ (unlike the foregoing approach, each firm $i$ has its own marginal cost $c_{i}$, besides its own production capacity $w_{i}$ ). With this oligopoly situation, there is associated the TU-game $\langle N, v\rangle$ given by $v(\emptyset):=0$ and (cf. [12], Lemma 4, page 194)

$$
v(S)=\frac{1}{4} \cdot\left[a-\min _{j \in S} c_{j}-w_{N \backslash S}\right]^{2} \quad \text { for all } S \subseteq N, S \neq \emptyset .
$$

In this framework, two fundamental assumptions do play a role, namely the expression $a-w_{N}$ is bounded above and below in the sense that $\max _{j \in N}\left[c_{j}-w_{j}\right] \leq a-w_{N} \leq \min _{j \in N}\left[c_{j}+w_{j}\right]$. Clearly, the lower bound (i.e., $a-w_{N} \geq c_{j}-w_{j}$ for all $j \in N$ ) implies that $a-\min _{j \in S} c_{j}-$ $w_{N \backslash S} \geq 0$ for all $S \subseteq N, S \neq \emptyset$. Due to this observation, (2.9) resembles (2.5). The oligopoly game $\langle N, v\rangle$ of $(2.9)$ is proven to be a convex game whenever all marginal costs are equal (i.e., $c_{j}=c_{k}$ for all $\left.j, k \in N\right)$. Necessary and sufficient (but extremely complicated) conditions for the convexity of this type of an oligopoly game are presented in [12] (Theorem 3 on page 195). Notice that our (common pool) model described by (2.1) is much more general than (2.9) since the average joint production function is not fixed at all (except to be weakly decreasing).

Example 2.6. Let the underlying joint production function $f$ be given by a bounded linear function, namely $f(x):=\min [\alpha \cdot x, \beta]$ for all $x \geq 0$, where $\alpha>0, \beta>0$. Thus, the average joint production function $h$ is a weakly decreasing function so that $h(x)=\alpha$ for all $0<x \leq \frac{\beta}{\alpha}$, and $h(x)=\frac{\beta}{x}$ for all $x \geq \frac{\beta}{\alpha}$. In the framework of a linear cost function $c(y):=c \cdot y$ for all $y \geq 0$ and a certain $0<c<\alpha$, we are able to determine the associated common pool game $\langle N, v\rangle$ of (2.1). We distinguish two cases concerning the sizes $w_{N}$ and $\frac{\beta}{\alpha}$.

Case one. Suppose $w_{N} \leq \frac{\beta}{\alpha}$. Since any feasible allocation $y \in\left[0, w_{S}\right]$ satisfies $y+w_{N \backslash S} \leq$ $w_{N} \leq \frac{\beta}{\alpha}$, we obtain that the maximization problem (2.1) with respect to coalition $S$ involves the linear objective function $y \cdot[\alpha-c]$. Its associated maximizer $y_{S}$ is given by $y_{S}=w_{S}$ and hence, $v(S)=w_{S} \cdot[\alpha-c]$ for all $S \subseteq N, S \neq \emptyset$. In words, if $w_{N} \leq \frac{\beta}{\alpha}$, then the common pool game $\langle N, v\rangle$ of (2.1) reduces to a so-called additive game (arising from the players' endowments) and thus, the convexity condition (1.4) holds clearly.

Case two. In the remainder suppose $w_{N}>\frac{\beta}{\alpha}$. Let $S \subseteq N, S \neq \emptyset$. In order to determine the worth $v(S)$, we have to compare the maximum of the first (linear) objective function $g_{1}(y):=y \cdot[\alpha-c]$ restricted to the (possibly empty) interval $\left[0, \frac{\beta}{\alpha}-w_{N \backslash S}\right]$ versus the second objective function $g_{2}(y):=y \cdot\left[\frac{\beta}{y+w_{N \backslash S}}-c\right]$ restricted to (the positive part of) the interval $\left[\frac{\beta}{\alpha}-w_{N \backslash S}, w_{S}\right]$. Firstly, note that $g_{1}(y) \leq g_{2}(y)$ for all $y \in\left[0, \frac{\beta}{\alpha}-w_{N \backslash S}\right]$ and further, $g_{1}(y)=g_{2}(y)$ for $y=\frac{\beta}{\alpha}-w_{N \backslash S}$. Secondly, note that the unconstrained maximization problem involving the second objective function $g_{2}(y)$ attains its maximum for $y=\gamma_{S}$, where $\gamma_{S}:=-w_{N \backslash S}+\sqrt{\frac{\beta \cdot w_{N \backslash S}}{c}}$ and its objective value equals $\gamma_{s} \cdot \sqrt{\frac{c}{w_{N \backslash S}}} \cdot\left[\sqrt{\beta}-\sqrt{c \cdot w_{N \backslash S}}\right]$.

Obviously, the relevant constraints $0 \leq \gamma_{S} \leq w_{S}$ and $\gamma_{S}+w_{N \backslash S} \geq \frac{\beta}{\alpha}$ respectively, are equivalent to the next constraints concerning the data $\alpha, \beta, c, w_{i}, i \in N$ :

$$
w_{N \backslash S} \leq \frac{\beta}{c} \quad \text { respectively } \quad w_{N \backslash S} \leq \frac{c \cdot\left(w_{N}\right)^{2}}{\beta} \quad \text { respectively } \quad w_{N \backslash S} \geq \frac{\beta \cdot c}{\alpha^{2}}
$$

Subcase 1. Suppose $w_{N \backslash S} \geq \frac{\beta}{c}$. Since $\alpha>c$, it follows that $y+w_{N \backslash S}>\frac{\beta}{\alpha}$ for all $y \geq 0$ and thus, the determination of $v(S)$ concerns only the second objective function $g_{2}(y)$ on 
$\left[0, w_{S}\right]$. Due to $\gamma_{S} \leq 0$ (because of the additional assumption $w_{N \backslash S} \geq \frac{\beta}{c}$ ), we conclude that the maximizer $y_{S}$ for the maximization problem (2.1) with respect to coalition $S$ is given by $y_{S}=0$ and thus, $v(S)=0$ whenever $w_{N \backslash S} \geq \frac{\beta}{c}$.

Subcase 2. Suppose $\frac{\beta \cdot c}{\alpha^{2}} \leq w_{N \backslash S} \leq \frac{\beta}{c}$. Since $\gamma_{S} \geq 0$ as well as $\gamma_{S} \geq \frac{\beta}{\alpha}-w_{N \backslash S}$, the objective function for the maximization problem (2.1) with respect to coalition $S$ is increasing on $\left[0, \gamma_{S}\right]$ and next decreasing. Hence, its maximizer $y_{S}$ is $\gamma_{S}$, provided $\gamma_{S} \leq w_{S}$. In other words, the maximizer $y_{S}$ equals $\gamma_{S}$ or $w_{S}$, whichever is less. Hence, $y_{S}=\min \left[\gamma_{S}, w_{S}\right]$ and thus, $v(S)=y_{S} \cdot\left[\frac{\beta}{y_{S}+w_{N \backslash S}}-c\right]$ whenever $\frac{\beta \cdot c}{\alpha^{2}} \leq w_{N \backslash S} \leq \frac{\beta}{c}$.

Subcase 3. Suppose $0 \leq w_{N \backslash S} \leq \frac{\beta \cdot c}{\alpha^{2}}$. By $\alpha>c$ and $w_{N}>\frac{\beta}{\alpha}$, we obtain $w_{N \backslash S}<\frac{\beta}{c}$ as well as $w_{N \backslash S}<\frac{c \cdot\left(w_{N}\right)^{2}}{\beta}$. That is, $0 \leq \gamma_{S}<w_{S}$ as well as $\gamma_{S} \leq \frac{\beta}{\alpha}-w_{N \backslash S}$. Thus, the objective function for the maximization problem (2.1) with respect to coalition $S$ is increasing on $\left[0, \frac{\beta}{\alpha}-w_{N \backslash S}\right]$ (due to its linear piece) and next decreasing. Hence, its maximizer $y_{S}$ equals $\frac{\beta}{\alpha}-w_{N \backslash S}$ and thus, $v(S)=\left[\frac{\beta}{\alpha}-w_{N \backslash S}\right] \cdot(\alpha-c)$ whenever $0 \leq w_{N \backslash S} \leq \frac{\beta \cdot c}{\alpha^{2}}$.

In summary, we conclude the following results:

$$
\begin{gathered}
y_{S}= \begin{cases}0 & \text { if } w_{N \backslash S} \geq \frac{\beta}{c}, \\
\min \left[\gamma_{S}, w_{S}\right] & \text { if } \frac{\beta \cdot c}{\alpha^{2}} \leq w_{N \backslash S} \leq \frac{\beta}{c}, \\
\frac{\beta}{\alpha}-w_{N \backslash S} & \text { if } 0 \leq w_{N \backslash S} \leq \frac{\beta \cdot c}{\alpha^{2}} .\end{cases} \\
v(S)= \begin{cases}0 & \text { if } w_{N \backslash S} \geq \frac{\beta}{c}, \\
y_{S} \cdot\left[\frac{\beta}{y_{S}+w_{N \backslash S}}-c\right] & \text { if } \frac{\beta \cdot c}{\alpha^{2}} \leq w_{N \backslash S} \leq \frac{\beta}{c}, \\
{\left[\frac{\beta}{\alpha}-w_{N \backslash S}\right] \cdot(\alpha-c)} & \text { if } 0 \leq w_{N \backslash S} \leq \frac{\beta \cdot c}{\alpha^{2}} .\end{cases}
\end{gathered}
$$

In spite of its complexity, the common pool game $\langle N, v\rangle$ of (2.11) turns out to be a convex game according to the theory developed in the next section concerning common pool games with respect to linear cost functions.

\section{The Common Pool Game: the case of a linear cost function}

In the context of the study on the convexity property for the common pool game, it turns out that the general framework needs additional (individually or jointly) assumptions about the cost function $c$ as well as the average joint production function $h$. For the special case with a linear cost function, however, the convexity of the common pool game will be established (without any further assumptions on either $c$ or $h$ ) by means of a tricky, but elegant proof. We start to present this elegant proof for this special case because it is extremely illustrative how to proceed with the more complicated treatment concerning the general case (with additional assumptions). Throughout the various forthcoming convexity proofs, we refer to the maximizer(s) for the maximization problem (2.1) with respect to different coalitions. Formally, for any $T \subseteq N, T \neq \emptyset$, let $y_{T} \in\left[0, w_{T}\right]$ be some maximizer for the maximization problem (2.1) with respect to coalition $T$ (provided it exists), that is $v(T)=y_{T} \cdot h\left(y_{T}+w_{N \backslash T}\right)-c\left(y_{T}\right)$. 
Theorem 3.1. Let $\langle N, v\rangle$ be the common pool game of (2.1) supposing the cost function $c$ is linear, i.e., $c(y):=c \cdot y$ for all $y \geq 0$ and a certain constant $c \geq 0$. That is,

$$
v(S):=\max _{0 \leq y \leq w_{S}}\left[y \cdot\left[h\left(y+w_{N \backslash S}\right)-c\right]\right] \quad \text { for all } S \subseteq N, S \neq \emptyset .
$$

Without any further assumption on the (weakly decreasing) average joint production function $h$, the common pool game $\langle N, v\rangle$ of (3.1) is a convex game (i.e., (1.4) holds).

Proof. Instead of the classical convexity condition (1.4), we prove the following equivalent convexity condition (based on the choice $T=S \cup\{j\}$ ):

$v(S \cup\{i\})-v(S) \leq v(S \cup\{i, j\})-v(S \cup\{j\}) \quad$ whenever $S \subseteq N \backslash\{i, j\}$ with $i \neq j$.

Let $i, j \in N, i \neq j$, and $S \subseteq N \backslash\{i, j\}$. Concerning the maximization problems (3.1) with respect to the two coalitions $S \cup\{i\}$ and $S \cup\{j\}$ respectively, we are interested in their maximizers $y_{S \cup\{i\}}$ and $y_{S \cup\{j\}}$ respectively in order to derive the following two equalities:

$$
\begin{aligned}
& v(S \cup\{i\})=y_{S \cup\{i\}} \cdot\left[h\left(y_{S \cup\{i\}}+w_{N \backslash(S \cup\{i\})}\right)-c\right] \\
& v(S \cup\{j\})=y_{S \cup\{j\}} \cdot\left[h\left(y_{S \cup\{j\}}+w_{N \backslash(S \cup\{j\})}\right)-c\right]
\end{aligned}
$$

Concerning the maximization problems (3.1) with respect to the two coalitions $S \cup\{i, j\}$ and $S$ respectively, we are interested in the feasible allocations $y_{S \cup\{j\}}+w_{i} \in\left[0, w_{S \cup\{i, j\}}\right]$ and $y_{S \cup\{i\}}-w_{i} \in\left[0, w_{S}\right]$ respectively in order to derive the following two inequalities:

$$
\begin{aligned}
v(S \cup\{i, j\}) & \geq\left[y_{S \cup\{j\}}+w_{i}\right] \cdot\left[h\left(y_{S \cup\{j\}}+w_{i}+w_{N \backslash(S \cup\{i, j\})}\right)-c\right] \\
v(S) & \geq\left[y_{S \cup\{i\}}-w_{i}\right] \cdot\left[h\left(y_{S \cup\{i\}}-w_{i}+w_{N \backslash S}\right)-c\right]
\end{aligned}
$$

Notice that $y_{S \cup\{j\}}+w_{i} \in\left[0, w_{S \cup\{i, j\}}\right]$ always holds, whereas $y_{S \cup\{i\}}-w_{i} \in\left[0, w_{S}\right]$ holds if and only if $y_{S \cup\{i\}} \geq w_{i}$. Moreover, (3.6) does not apply at all whenever $S=\emptyset$. By (3.3)-(3.6), together with the common relationship $w_{N \backslash T}=w_{k}+w_{N \backslash(T \cup\{k\})}$ whenever $T \subseteq N \backslash\{k\}$, we arrive at the following two inequalities:

$$
\begin{array}{r}
v(S \cup\{i, j\})-v(S \cup\{j\}) \\
\geq w_{i} \cdot\left[h\left(y_{S \cup\{j\}}+w_{N \backslash(S \cup\{j\})}\right)-c\right] \\
v(S \cup\{i\})-v(S) \leq w_{i} \cdot\left[h\left(y_{S \cup\{i\}}+w_{N \backslash(S \cup\{i\})}\right)-c\right]
\end{array}
$$

where the latter inequality (3.8) is valid only if $y_{S \cup\{i\}} \geq w_{i}$ and $S \neq \emptyset$. Clearly, the alternative convexity condition (3.2) is a direct consequence of both inequalities (3.7)-(3.8) as soon as the weakly decreasing function $h$ satisfies the next condition:

$$
\begin{array}{rlr}
h\left(y_{S \cup\{j\}}+w_{N \backslash(S \cup\{j\})}\right) & \geq h\left(y_{S \cup\{i\}}+w_{N \backslash(S \cup\{i\})}\right) & \text { or equivalently, } \\
y_{S \cup\{j\}}+w_{N \backslash(S \cup\{j\})} & \leq y_{S \cup\{i\}}+w_{N \backslash(S \cup\{i\})} & \text { or equivalently, } \\
y_{S \cup\{j\}}-w_{j} & \leq y_{S \cup\{i\}}-w_{i} &
\end{array}
$$


In fact, the latter inequality (3.10) may be considered as an assumption, without loss of generality, because the roles of both players $i$ and $j$ in the alternative convexity condition (3.2) are interchangeable (that is, the relevant condition (3.2) does not change at all by interchanging the two players). In other words, in case the assumption $y_{S \cup\{j\}}-w_{j} \leq y_{S \cup\{i\}}-$ $w_{i}$ is not valid (or equivalently, $y_{S \cup\{i\}}-w_{i} \leq y_{S \cup\{j\}}-w_{j}$ ), then an identical proof applies in which player $i$ takes the role of player $j$ (and vice versa). This completes the proof of the exact outline of the alternative convexity condition (3.2), provided $y_{S \cup\{i\}} \geq w_{i}$ and $S \neq \emptyset$. It remains to establish the proof of (3.2) whenever $0 \leq y_{S \cup\{i\}} \leq w_{i}$. Note that the remaining case covers the subcase $S=\emptyset$ too because of $0 \leq y_{\{i\}} \leq w_{i}$. Moreover, the general inequalities (3.7) and (3.9) are still valid.

¿From (3.7), (3.9) and the assumption $0 \leq y_{S \cup\{i\}} \leq w_{i}$ respectively, we conclude that the following chain of inequalities holds:

$$
\begin{aligned}
v(S \cup\{i, j\})-v(S \cup\{j\}) & \geq w_{i} \cdot\left[h\left(y_{S \cup\{j\}}+w_{N \backslash(S \cup\{j\})}\right)-c\right] \\
& \geq w_{i} \cdot\left[h\left(y_{S \cup\{i\}}+w_{N \backslash(S \cup\{i\})}\right)-c\right] \\
& \geq y_{S \cup\{i\}} \cdot\left[h\left(y_{S \cup\{i\}}+w_{N \backslash(S \cup\{i\})}\right)-c\right] \\
& =v(S \cup\{i\}) \geq v(S \cup\{i\})-v(S)
\end{aligned}
$$

where the latter inequality is due to the fact that $v(S) \geq 0$ ( such that $v(S)=0$ if $S=\emptyset$ ). This completes the full proof of the convexity property (3.2) for the common pool game assuming a linear cost function.

\section{Convexity of the Common Pool Game: the general case}

This section aims to introduce the weakest form of any conditions (involving the cost function $c$ as well as the average joint production function $h$ ) that are sufficient to guarantee the convexity of the common pool game. Concerning the symmetric case (in which all the players are supposed to possess identical endowments), we claim that it suffices for both the marginal costs and the average costs to be weakly decreasing (e.g., $c(x):=\sqrt{x}$ ). Generally speaking, we claim that, for convexity of the common pool game, it suffices that marginal costs are weakly decreasing in such a way that the difference of two marginal costs is bounded above by some marginal returns of the average joint production function. For the case with a linear cost function, the latter condition fully agrees with the assumption that $h$ is a weakly decreasing function. Before we start to prove these two main results, we recall that the common pool game $\langle N, v\rangle$ of (2.1) is a monotonic game, i.e., $v(S \cup\{i\}) \geq v(S)$ for all $i \in N$ and all $S \subseteq N \backslash\{i\}$. Particularly, if $w_{i}=0$, then $v(S \cup\{i\})=v(S)$ for all $S \subseteq N \backslash\{i\}$.

Definition 4.1. Consider a common pool situation with players' endowments $w_{i} \geq 0, i \in N$, the cost function $c: \mathbb{R}_{+} \rightarrow \mathbb{R}_{+}$satisfying $c(0)=0$ as well as the average joint production function $h: \mathbb{R}_{+} \rightarrow \mathbb{R}_{+}$. The derivative $\Delta c: \mathbb{R}_{+} \times \mathbb{R}_{++} \rightarrow \mathbb{R}$ of the cost function $c$ is given by $(\Delta c)(x, \epsilon):=\frac{c(x+\epsilon)-c(x)}{\epsilon}$ for all $x \geq 0$ and all $\epsilon>0$. With every coalition $T \subseteq N, T \neq \emptyset$, there is associated the net-benefit function $b_{T}: \mathbb{R}_{+} \times \mathbb{R}_{++} \rightarrow \mathbb{R}$ given by

$$
b_{T}(x, \epsilon):=h\left(x+\epsilon+w_{N \backslash T}\right)-(\Delta c)(x, \epsilon) \quad \text { for all } x \geq 0 \text { and all } \epsilon>0 .
$$


In words, $b_{T}(x, \epsilon)$ represents the net-benefit for coalition $T$ whenever the initial production level $x$ is increased by the amount of $\epsilon$, assuming that any member $j$ of the complementary coalition $N \backslash T$ invests the full individual endowment $w_{j}$. For any $T \subseteq N, T \neq \emptyset$, recall that $y_{T} \in\left[0, w_{T}\right]$ denotes some maximizer for the maximization problem $(2.1)$ with respect to coalition $T$.

Theorem 4.2. The common pool game $\langle N, v\rangle$ of (2.1) is a convex game (i.e., (1.4) holds) whenever the cost function $c$ and the various net-benefit functions $b_{T}, T \subseteq N, T \neq \emptyset$, satisfy the following two conditions (4.2) and (4.3) respectively:

(i) The cost derivative $\Delta c$ (or equivalently, the marginal cost function) is weakly decreasing, that is

$$
(\Delta c)(x, \epsilon) \geq(\Delta c)(y, \epsilon) \quad \text { for all } y \geq x \geq 0 \text { and all } \epsilon>0
$$

(ii) The net-benefit functions are weakly increasing with respect to the inclusion of sets, that is

$$
b_{S \cup\{i, j\}}\left(y_{S \cup\{j\}}, w_{i}\right) \geq b_{S \cup\{i\}}\left(y_{S \cup\{i\}}-w_{i}, w_{i}\right)
$$

for all $i, j \in N, i \neq j$, and all $S \subseteq N \backslash\{i, j\}, S \neq \emptyset$, with $y_{S \cup\{i\}} \geq w_{i}>0$.

Remark 4.3. Concerning the sequential process of the formation of the grand coalition $N$, any player $i$ can join any coalition $T \subseteq N \backslash\{i\}$ and produce a net-benefit $b_{T \cup\{i\}}\left(z, w_{i}\right)$ by investing the individual endowment $w_{i}$ additional to the initial production level $z$ of coalition $T$. In this setting, the fundamental condition (4.3) requires that the larger the coalition to which a player joins, the higher the enlarged coalition's net-benefit, taken into account certain (optimal or feasible) production levels for the smaller and larger coalition respectively. Thus, (4.3) guarantees that there exist strong incentives for mutual cooperation and so, these strong preferences for the formation of the grand coalition do overcome the tragedy of the commons (in the framework of common pool situations), as has been mentioned by [4].

Remark 4.4. Condition (4.3) will be simplified, but strengthened if the (unknown) maximizers are replaced by arbitrary real numbers as follows. For notation' sake, write $x_{1}:=$ $y_{S \cup\{j\}}-w_{j}, x_{2}:=y_{S \cup\{i\}}-w_{i}, z:=w_{N \backslash S}$, and $\epsilon_{1}:=w_{i}, \epsilon_{2}:=w_{j}$. Now (4.3) will be strengthened to the next fundamental condition:

$$
h\left(x_{1}+z\right)-h\left(x_{2}+z\right) \geq(\Delta c)\left(x_{1}+\epsilon_{2}, \epsilon_{1}\right)-(\Delta c)\left(x_{2}, \epsilon_{1}\right)
$$

whenever $x_{2} \geq 0, x_{1}+z \geq 0, \epsilon_{1}>0$ and $\epsilon_{2}>0$. Without loss of generality, we may assume $x_{1} \leq x_{2}$ (because the roles of both players $i$ and $j$ in the alternative convexity condition (3.2) are interchangeable). Notice that, if the cost function $c$ is linear, then (4.4) reduces to $h\left(x_{1}+z\right) \geq h\left(x_{2}+z\right)$ whenever $x_{1}+z \leq x_{2}+z$, which result holds true since $h$ is a weakly decreasing function. Moreover, (4.4) applied to $x_{1}=x_{2}$ reduces to the inequality $(\Delta c)\left(x_{1}, \epsilon_{1}\right) \geq(\Delta c)\left(x_{1}+\epsilon_{2}, \epsilon_{1}\right)$ for all $x_{1} \geq 0$, all $\epsilon_{1}>0$, and all $\epsilon_{2}>0$, that is, the marginal costs are weakly decreasing. We conclude that the concavity of the cost function $c$ (i.e., $\left.c^{\prime \prime} \leq 0\right)$ is a desirable property, together with (4.4). Under the additional (but not necessary) assumption that the cost function $c$ is convex (i.e., $c^{\prime \prime} \geq 0$ ), then the only remaining possible cost function is the linear one as studied in the previous section. 
In summary, the common pool game $\langle N, v\rangle$ of (2.1) is a convex game whenever the (weakly decreasing) cost derivative function $\Delta c$ and the (weakly decreasing) average joint production function $h$ satisfy the mutual condition (4.4). In words, the boundedness above of the difference of two marginal costs by a particular marginal return of the average joint production function is sufficient for the convexity of the common pool game.

Corollary 4.5. Consider the symmetric case in that all the players possess identical endowments, i.e., suppose $w_{i}:=w$ for all $i \in N$. The symmetrical common pool game $\langle N, v\rangle$ of (2.1) is a convex game (i.e., (1.4) holds) whenever the cost function $c$ has both weakly decreasing marginal costs and weakly decreasing average costs respectively in the following sense:

$$
\begin{aligned}
c(x)-c(x-w) & \geq c(x+w)-c(x) \quad \text { for all } x \geq w \text { and } \\
\frac{c(x+w)}{x+w} & \leq \frac{c(x)}{x} \quad \text { for all } 0<x \leq w . \quad \text { Example: } c(x):=\sqrt{x}
\end{aligned}
$$

Proof of Theorem 4.2. Instead of the classical convexity condition (1.4), we prove the alternative convexity condition (3.2), that is

$$
v(S \cup\{i\})-v(S) \leq v(S \cup\{i, j\})-v(S \cup\{j\}) \quad \text { whenever } S \subseteq N \backslash\{i, j\} \text { with } i \neq j .
$$

Let $i, j \in N, i \neq j$, and $S \subseteq N \backslash\{i, j\}$. Notice that the roles of both players $i$ and $j$ in the alternative convexity condition (3.2) are interchangeable. If $w_{i}=0$ or $w_{j}=0$, then the inequality in (3.2) is an equality because $i$ or $j$ respectively is a dummy player. If $v(S \cup\{i\})=0$, then the inequality in (3.2) becomes trivial due to the monotonicity of the common pool game (yielding $v(S)=0$ as well as $v(S \cup\{i, j\}) \geq v(S \cup\{j\})$ ). Thus, in the remainder, we may suppose, without loss of generality, that the endowments and the maximizers satisfy both $w_{i}>0, w_{j}>0$ and $y_{S \cup\{i\}}>0, y_{S \cup\{j\}}>0$.

Concerning the maximization problems (2.1) with respect to the two coalitions $S \cup\{i\}$ and $S \cup\{j\}$ respectively, we are interested in their maximizers $y_{S \cup\{i\}}$ and $y_{S \cup\{j\}}$ respectively in order to derive the following two equalities:

$$
\begin{aligned}
& v(S \cup\{i\})=y_{S \cup\{i\}} \cdot h\left(y_{S \cup\{i\}}+w_{N \backslash(S \cup\{i\})}\right)-c\left(y_{S \cup\{i\}}\right) \\
& v(S \cup\{j\})=y_{S \cup\{j\}} \cdot h\left(y_{S \cup\{j\}}+w_{N \backslash(S \cup\{j\})}\right)-c\left(y_{S \cup\{j\}}\right)
\end{aligned}
$$

Concerning the maximization problems (2.1) with respect to the two coalitions $S \cup\{i, j\}$ and $S$ respectively, we are interested in the feasible allocations $y_{S \cup\{j\}}+w_{i} \in\left[0, w_{S \cup\{i, j\}}\right]$ and $y_{S \cup\{i\}}-w_{i} \in\left[0, w_{S}\right]$ respectively in order to derive the following two inequalities:

$$
\begin{aligned}
v(S \cup\{i, j\}) & \geq\left[y_{S \cup\{j\}}+w_{i}\right] \cdot h\left(y_{S \cup\{j\}}+w_{i}+w_{N \backslash(S \cup\{i, j\})}\right)-c\left(y_{S \cup\{j\}}+w_{i}\right) \\
v(S) & \geq\left[y_{S \cup\{i\}}-w_{i}\right] \cdot h\left(y_{S \cup\{i\}}-w_{i}+w_{N \backslash S}\right)-c\left(y_{S \cup\{i\}}-w_{i}\right)
\end{aligned}
$$

Notice that $y_{S \cup\{j\}}+w_{i} \in\left[0, w_{S \cup\{i, j\}}\right]$ always holds, whereas $y_{S \cup\{i\}}-w_{i} \in\left[0, w_{S}\right]$ holds if and only if $y_{S \cup\{i\}} \geq w_{i}$. Moreover, (4.8) does not apply at all whenever $S=\emptyset$. By (4.5)-(4.8), together with the common relationship $w_{N \backslash T}=w_{k}+w_{N \backslash(T \cup\{k\})}$ whenever $T \subseteq N \backslash\{k\}$, we arrive at the following two inequalities:

$$
\begin{aligned}
v(S \cup\{i, j\})-v(S \cup\{j\}) & \geq w_{i} \cdot h\left(y_{S \cup\{j\}}+w_{N \backslash(S \cup\{j\})}\right)+c\left(y_{S \cup\{j\}}\right)-c\left(y_{S \cup\{j\}}+w_{i}\right) \\
v(S \cup\{i\})-v(S) & \leq w_{i} \cdot h\left(y_{S \cup\{i\}}+w_{N \backslash(S \cup\{i\})}\right)+c\left(y_{S \cup\{i\}}-w_{i}\right)-c\left(y_{S \cup\{i\}}\right)(
\end{aligned}
$$


where the latter inequality (4.10) is valid only if $y_{S \cup\{i\}} \geq w_{i}$ and $S \neq \emptyset$. Clearly, in order to deduce the alternative convexity condition (3.2) directly from both inequalities (4.9)-(4.10), the weakest form of any condition is that both functions $h$ and $c$ satisfy the next condition:

$h\left(y_{S \cup\{j\}}+w_{N \backslash(S \cup\{j\})}\right)-(\Delta c)\left(y_{S \cup\{j\}}, w_{i}\right) \geq h\left(y_{S \cup\{i\}}+w_{N \backslash(S \cup\{i\})}\right)-(\Delta c)\left(y_{S \cup\{i\}}-w_{i}, w_{i}\right)(4.11)$ provided $y_{S \cup\{i\}} \geq w_{i}$ and $S \neq \emptyset$. Clearly, (4.11) is fully equivalent to (4.3).

Finally, it remains to prove the alternative convexity condition (3.2) in the degenerated case $0 \leq y_{S \cup\{i\}} \leq w_{i}$. Note that the remaining case covers the subcase $S=\emptyset$ too because of $0 \leq y_{\{i\}} \leq w_{i}$. Moreover, the general inequalities (4.9) and the assumption (3.9) are still valid.

¿From (4.9), (3.9) and the assumption $0<y_{S \cup\{i\}} \leq w_{i}$ respectively, we conclude that the following chain of (in)equalities holds:

$$
\begin{aligned}
& v(S \cup\{i, j\})-v(S \cup\{j\}) \\
\geq & w_{i} \cdot h\left(y_{S \cup\{j\}}+w_{N \backslash(S \cup\{j\})}\right)-\left[c\left(y_{S \cup\{j\}}+w_{i}\right)-c\left(y_{S \cup\{j\}}\right)\right] \\
\geq & w_{i} \cdot h\left(y_{S \cup\{i\}}+w_{N \backslash(S \cup\{i\})}\right)-\left[c\left(y_{S \cup\{j\}}+w_{i}\right)-c\left(y_{S \cup\{j\}}\right)\right] \\
= & w_{i} \cdot\left[h\left(y_{S \cup\{i\}}+w_{N \backslash(S \cup\{i\})}\right)-\frac{c\left(y_{S \cup\{j\}}+w_{i}\right)-c\left(y_{S \cup\{j\}}\right)}{w_{i}}\right] \\
\geq & y_{S \cup\{i\}} \cdot\left[h\left(y_{S \cup\{i\}}+w_{N \backslash(S \cup\{i\})}\right)-\frac{c\left(y_{S \cup\{j\}}+w_{i}\right)-c\left(y_{S \cup\{j\}}\right)}{w_{i}}\right] \\
= & v(S \cup\{i\})+c\left(y_{S \cup\{i\}}\right)-y_{S \cup\{i\}} \cdot\left[\frac{c\left(y_{S \cup\{j\}}+w_{i}\right)-c\left(y_{S \cup\{j\}}\right)}{w_{i}}\right] \\
\geq & v(S \cup\{i\}) \geq v(S \cup\{i\})-v(S)
\end{aligned}
$$

where the last inequality but one reduces to the following inequality:

$$
\frac{c\left(y_{S \cup\{i\}}\right)}{y_{S \cup\{i\}}} \geq \frac{c\left(y_{S \cup\{j\}}+w_{i}\right)-c\left(y_{S \cup\{j\}}\right)}{w_{i}} \quad \text { given that } 0<y_{S \cup\{i\}} \leq w_{i} .
$$

Since the marginal cost function is supposed to be weakly decreasing, together with the assumption $0<y_{S \cup\{i\}} \leq w_{i}$, and the fact that the average cost function $\frac{c(x)}{x}$ is weakly decreasing (by concavity of $c$ ), we conclude that the following chain of inequalities holds:

$$
\frac{c\left(y_{S \cup\{j\}}+w_{i}\right)-c\left(y_{S \cup\{j\}}\right)}{w_{i}} \leq \frac{c\left(0+w_{i}\right)-c(0)}{w_{i}}=\frac{c\left(w_{i}\right)}{w_{i}} \leq \frac{c\left(y_{S \cup\{i\}}\right)}{y_{S \cup\{i\}}}
$$

This proves the claim (4.12) and thus, this completes the proof of the alternative convexity condition (3.2) in the degenerated case $0<y_{S \cup\{i\}} \leq w_{i}$. This proves Theorem 4.2.

Proof of Corollary 4.5. Suppose $w_{i}:=w$ for all $i \in N$. Clearly, for any $T \subseteq N, T \neq \emptyset$, the maximizer $y_{T}$ for the maximization problem (2.1) with respect to coalition $T$ depends not anymore on the players in $T$, but only on the coalition size. Thus, $y_{S \cup\{i\}}=y_{S \cup\{j\}}$ for all $i, j \in N, i \neq j$, and $S \subseteq N \backslash\{i, j\}$. Consequently, in the previous proof, the first fundamental 
condition (4.11) reduces to the following condition: $c(x)-c(x-w) \geq c(x+w)-c(x)$ whenever $x \geq w$. Moreover, the second fundamental condition (4.12) reduces to the following condition:

$$
\frac{c(x)}{x} \geq \frac{c(x+w)-c(x)}{w} \quad \text { or equivalently, } \quad \frac{c(x+w)}{x+w} \leq \frac{c(x)}{x} \quad \text { whenever } 0<x \leq w .
$$

This proves Corollary 4.5.

\section{Average-Convexity of the general Common Pool Game}

As shown by Proposition 2.4, the common pool game with a general cost function may fail to be convex. In this section our main goal is to investigate a certain relaxation of the convexity condition known as the average-convexity condition. The common pool TU-game $\langle N, v\rangle$ is said to be average-convex if its characteristic function $v: \mathcal{P}(N) \rightarrow \mathbb{R}$, as given by (2.1), satisfies the following condition (cf. [5]):

$$
\sum_{i \in S}[v(S)-v(S \backslash\{i\})] \leq \sum_{i \in S}[v(T)-v(T \backslash\{i\})] \quad \text { for all } S \subseteq T \subseteq N, S \neq \emptyset .
$$

Note that convex games satisfy the average-convexity condition (by summing up, over all $i \in S$, the valid convexity conditions $v(S)-v(S \backslash\{i\}) \leq v(T)-v(T \backslash\{i\}))$. The main theorem states the weakest form of any conditions (involving weighted sums of the various net-benefit functions $b_{T}, T \subseteq N, T \neq \emptyset$; see Definition 4.1) that are sufficient to guarantee the averageconvexity of the common pool game.

Theorem 5.1. The common pool game $\langle N, v\rangle$ of (2.1) is an average-convex game (i.e., (5.1) holds) whenever the endowments $w_{i}, i \in N$, and the various net-benefit functions $b_{T}, T \subseteq N$, $T \neq \emptyset$, satisfy the next mutual condition: for all $\emptyset \neq S \subsetneq T \subseteq N$ with $y_{S}>0$ it holds

$$
\sum_{i \in S} w_{i} \cdot b_{T}\left(y_{T \backslash\{i\}}, w_{i}\right) \geq \sum_{\substack{i \in S, w_{i} \leq y_{S}}} w_{i} \cdot b_{S}\left(y_{S}-w_{i}, w_{i}\right)+b_{S}\left(0, y_{S}\right) \cdot \sum_{\substack{i \in S, w_{i} \geq y_{S}}} w_{i}
$$

The technical and tedious proof will be omitted, but is available upon request (cf. [2]).

Remark 5.2. In the setting of the sequential process of the formation of the grand coalition $N$, condition (5.2) requires that the weighted sum of net-benefits of members of a coalition $S$ with respect to the formation of a superset $T$ is at least as much as the one with respect to the formation of the coalition itself. The latter weighted sum is decomposed into two types of a weighted sum since every member $i$ of coalition $S$ invests his individual endowment $w_{i}$ (to contribute to the joint optimal production level $y_{S}$ of coalition $S$ ) or invests the optimal production level $y_{S}$ himself, whichever is less.

In case of a linear cost function $c$, condition (5.2) reduces to

$$
\sum_{i \in S} w_{i} \cdot h\left(y_{T \backslash\{i\}}+w_{i}+w_{N \backslash T}\right) \geq h\left(y_{S}+w_{N \backslash S}\right) \cdot \sum_{i \in S} w_{i} \quad \text { for all } \emptyset \neq S \varsubsetneqq T \subseteq N .
$$

In fact, we claim that every separate term $h\left(y_{T \backslash\{i\}}+w_{i}+w_{N \backslash T}\right)-h\left(y_{S}+w_{N \backslash S}\right), i \in S$, is non-negative. For that purpose, because $h$ is a weakly decreasing function, it suffices to check that $y_{T \backslash\{i\}}+w_{i}+w_{N \backslash T} \leq y_{S}+w_{N \backslash S}$ or equivalently, $y_{T \backslash\{i\}}+w_{i} \leq y_{S}+w_{T \backslash S}$ 
whenever $\emptyset \neq S \varsubsetneqq T \subseteq N$ and $i \in S$. In case $T=S \cup\{j\}$ for some $j \in T \backslash S$, then the relevant inequality $y_{T \backslash\{i\}}+w_{i} \leq y_{T \backslash\{j\}}+w_{j}$ (where $i \in S, j \notin S$ ) may be treated as an assumption based on the interchangeability of the two players $i, j$ with respect to the expression $[v(S \cup\{j\})-v((S \cup\{j\}) \backslash\{i\})]-[v(S)-v(S \backslash\{i\})]$ or equivalently, $[v(S \cup\{j\})-v(S)]-$ $[v((S \cup\{j\}) \backslash\{i\})-v(S \backslash\{i\})]$. In case $T \backslash S$ contains at least two players, then the inequality $y_{T \backslash\{i\}}+w_{i} \leq y_{S}+w_{T \backslash S}$ follows by a similar argument. Particularly, these observations establish the average-convexity of common pool games with respect to linear cost functions, although the proof of the average convexity through (5.2) is much more difficult to complete in comparison to its convexity proof as treated in Section 3.

\section{References}

[1] Driessen, T.S.H., (1988), Cooperative Games, Solutions, and Applications. Kluwer Academic Publishers, Dordrecht, The Netherlands.

[2] Driessen, T.S.H. and H. Meinhardt, (2000), Convexity and average convexity of common pool TU-games. Memorandum, Faculty of Mathematical Sciences, University of Twente, Enschede, The Neherlands.

[3] Funaki, Y. and T. Yamato, (1999), The core of an economy with a common pool resource: a partition function form approach. International Journal of Game Theory 28, 157-171.

[4] Hardin, G., (1968), The tragedy of the commons. Science 162, 1243-1248.

[5] Iñarra, E. and J.M. Usategui, (1993), The Shapley value and average convex games. International Journal of Game Theory 22, 13-29.

[6] Meinhardt, H., (1999), Common pool games are convex games. Journal of Public Economic Theory 2, 247-270.

[7] Meinhardt, H., (1999), Convexity and k-convexity in cooperative common pool games. Discussion Paper 11, Institute for Statistics and Economic Theory, University of Karlsruhe, Karlsruhe, Germany.

[8] Meinhardt, H., (2000), Incentives for cooperative decision making in common pool situations. Ph.D. thesis, Department of Economics, University of Karlsruhe, Karlsruhe, Germany.

[9] Roemer, J., (1989), Public ownership resolution of the tragedy of the commons. Social Philosophy and Policy 6, 74-92.

[10] Shapley, L.S., (1971), Cores of convex games. International Journal of Game Theory 1, $11-26$.

[11] Weitzman, M.L., (1974), Free access vs. private ownership as alternative systems for managing common property. Journal of Economic Theory 8, 225-234.

[12] Zhao, J., (1999), A necessary and sufficient condition for the convexity in oligopoly games. Mathematical Social Sciences 37, 189-204. 


\section{APPENDIX: the Proof of Theorem 5.1}

Theorem 6.1. The common pool game $\langle N, v\rangle$ of (2.1) is an average-convex game (i.e., (5.1) holds) whenever the endowments $w_{i}, i \in N$, and the various net-benefit functions $b_{T}, T \subseteq N$, $T \neq \emptyset$, satisfy the next mutual condition: for all $\emptyset \neq S \varsubsetneqq T \subseteq N$ with $y_{S}>0$ it holds

$$
\sum_{i \in S} w_{i} \cdot b_{T}\left(y_{T \backslash\{i\}}, w_{i}\right) \geq \sum_{\substack{i \in S, w_{i} \leq y_{S}}} w_{i} \cdot b_{S}\left(y_{S}-w_{i}, w_{i}\right)+b_{S}\left(0, y_{S}\right) \cdot \sum_{\substack{i \in S, w_{i} \geq y_{S}}} w_{i}
$$

Proof. To start with, recall that the classical convexity condition (1.4) has been replaced by the equivalent convexity condition (3.2) (due to the choice $T=S \cup\{j\}$ ), in which condition the roles of both players $i$ and $j$ are interchangeable. A similar simplification (based on the choice $T=S \cup\{j\}$ ) is not applicable to the average-convexity condition (5.1) since the equivalence of the two resulting conditions turns out to be lost. Moreover, the interchangeable roles of two players is lost too because the average-convexity condition (5.1) deals with the marginal contributions of all the players in any coalition.

In order to investigate the average-convexity property for the common pool game $\langle N, v\rangle$ of (2.1), let $S \varsubsetneqq T \subseteq N$ where $S \neq \emptyset$. Concerning the maximization problems (2.1) with respect to the coalitions $S$ and $T \backslash\{i\}, i \in S$, respectively, we are interested in their maximizers $y_{S}$ and $y_{T \backslash\{i\}}, i \in S$, respectively in order to derive the following equalities:

$$
\begin{aligned}
v(S) & =y_{S} \cdot h\left(y_{S}+w_{N \backslash S}\right)-c\left(y_{S}\right) \\
v(T \backslash\{i\}) & =y_{T \backslash\{i\}} \cdot h\left(y_{T \backslash\{i\}}+w_{N \backslash(T \backslash\{i\})}\right)-c\left(y_{T \backslash\{i\}}\right) \quad \text { for every } i \in S .
\end{aligned}
$$

If $y_{s}=0$, then $v(S)=0$ and the inequality in (5.1) becomes trivial due to the monotonicity of the common pool game (yielding $v(S \backslash\{i\})=0$ for all $i \in S$ as well as $v(T) \geq v(T \backslash\{i\}$ ) for all $i \in S$ ). Thus, without loss of generality, suppose $y_{S}>0$.

Notice that $T \backslash\{i\} \neq \emptyset$ for all $i \in S$ since $T$ contains at least two players. Concerning the maximization problem (2.1) with respect to coalition $T$, we are interested, for every $i \in S$, in the feasible allocations $y_{T \backslash\{i\}}+w_{i} \in\left[0, w_{T}\right]$ respectively in order to derive the following system of inequalities:

$$
v(T) \geq\left[y_{T \backslash\{i\}}+w_{i}\right] \cdot h\left(y_{T \backslash\{i\}}+w_{i}+w_{N \backslash T}\right)-c\left(y_{T \backslash\{i\}}+w_{i}\right) \quad \text { for every } i \in S .
$$

By (6.3)-(6.4), together with the common relationship $\left.w_{N \backslash(T \backslash\{i\})}\right)=w_{i}+w_{N \backslash T}$ whenever $i \in T$, we arrive at the following system of inequalities: for all $i \in S$

$$
\begin{gathered}
v(T)-v(T \backslash\{i\}) \geq w_{i} \cdot h\left(y_{T \backslash\{i\}}+w_{i}+w_{N \backslash T}\right)-\left[c\left(y_{T \backslash\{i\}}+w_{i}\right)-c\left(y_{T \backslash\{i\}}\right)\right] \\
\text { or equivalently, } \quad v(T)-v(T \backslash\{i\}) \geq w_{i} \cdot b_{T}\left(y_{T \backslash\{i\}}, w_{i}\right) \quad \text { for all } i \in S .
\end{gathered}
$$

(cf. (4.1)). By summing up (6.5) over all $i \in S$, we obtain the following inequality:

$$
\sum_{i \in S}[v(T)-v(T \backslash\{i\})] \geq \sum_{i \in S} w_{i} \cdot b_{T}\left(y_{T \backslash\{i\}}, w_{i}\right)
$$

Concerning the maximization problems (2.1) with respect to the various coalitions $S \backslash\{i\}$, $i \in S$, we are interested, for every $i \in S$, in the feasible allocations $y_{S}-w_{i} \in\left[0, w_{S \backslash\{i\}}\right]$ respectively in order to derive the following system of inequalities:

$$
v(S \backslash\{i\}) \geq\left[y_{S}-w_{i}\right] \cdot h\left(y_{S}-w_{i}+w_{N \backslash(S \backslash\{i\})}\right)-c\left(y_{S}-w_{i}\right) \quad \text { provided } y_{S} \geq w_{i} .
$$


By (6.2) and (6.7), we arrive, for every $i \in S$ satisfying $y_{S} \geq w_{i}$, at the following inequality:

$$
\begin{aligned}
& v(S)-v(S \backslash\{i\}) \leq w_{i} \cdot h\left(y_{S}+w_{N \backslash S}\right)-\left[c\left(y_{S}\right)-c\left(y_{S}-w_{i}\right)\right] \quad \text { or equivalently, } \\
& v(S)-v(S \backslash\{i\}) \leq w_{i} \cdot b_{S}\left(y_{S}-w_{i}, w_{i}\right) \quad \text { for all } i \in S \text { satisfying } y_{S} \geq w_{i} .
\end{aligned}
$$

In case $0<y_{S} \leq w_{i}$ (e.g. if coalition $S$ consists of an individual), then we conclude that the following chain of (in)equalities holds:

$$
\begin{aligned}
v(S)-v(S \backslash\{i\}) & \leq v(S) & & (\text { since } v(S \backslash\{i\}) \geq 0) \\
& =y_{S} \cdot\left[h\left(y_{S}+w_{N \backslash S}\right)-\frac{c\left(y_{S}\right)}{y_{S}}\right] & & (\text { by }(6.2)) \\
& \leq w_{i} \cdot\left[h\left(y_{S}+w_{N \backslash S}\right)-\frac{c\left(y_{S}\right)}{y_{S}}\right] & & \text { (by assumption of } \left.y_{S} \leq w_{i}\right)
\end{aligned}
$$

and consequently,

$$
v(S)-v(S \backslash\{i\}) \leq w_{i} \cdot b_{S}\left(0, y_{S}\right) \quad \text { for all } i \in S \text { satisfying } 0<y_{S} \leq w_{i} .
$$

By summing up (6.8) and (6.9) over all $i \in S$, we obtain the following inequality:

$$
\sum_{i \in S}[v(S)-v(S \backslash\{i\})] \leq \sum_{\substack{i \in S, w_{i} \leq y_{S}}} w_{i} \cdot b_{S}\left(y_{S}-w_{i}, w_{i}\right)+b_{S}\left(0, y_{S}\right) \cdot \sum_{\substack{i \in S, w_{i} \geq y_{S}}} w_{i}
$$

Clearly, in order to deduce the average-convexity condition (5.1) directly from both inequalities (6.6) and (6.10), the weakest form of any condition is given by (6.1) (provided $\emptyset \neq S \varsubsetneqq T \subseteq N$ and $\left.y_{S}>0\right)$. This completes the proof of Theorem 5.1. 